\title{
Recurrent Stroke as the First Manifestation in a Patient Infected with HIV- A Case Report
}

Hegde SS ${ }^{1 *}$, Muhammed Ismail ${ }^{1}$, KulamarvaRama $\mathbf{S}^{1}$ and HulegarAbhishek ${ }^{2}$

${ }^{1}$ Kasturba Medical College, Mangalore, India

${ }^{2}$ Bangalore Medical College, Bangalore, India

\begin{abstract}
This case involves a 26 -year-old, HIV-infected woman who presented with recurrent ischemic strokes as the first manifestation of HIV infection. The patient reported a history of three episodes of stroke in the previous 10 years. Recent admission presentation was of left hemiparesis and pseudobulbar palsy. She underwent an extensive workup during her first stroke episode for the risk factors associated with stroke in a very young woman, but no cause found other than positive HIV-1 antibodies. Contrast CT scan of the brain revealed vasculitic changes, but again only HIV found. This case illustrates the possibility of HIV manifesting first as recurrent stroke.
\end{abstract}

Keywords: Ischaemic; Stroke; HIV; Recurrent

\section{Background}

The literature describes an increased risk of stroke in HIV patients [1]. Etiological factors implicated in the occurrence of stroke in HIV include opportunistic infections such as Mycobacterium tuberculosis, $\mathrm{CMV}, \mathrm{HZV}$, toxoplasmosis, pneumocystis, salmonella, syphilis, cryptococcosis and other mycoses; neoplasia including lymphoma, HIV induced cardiac disease, HIV associated cerebral vasculopathy, prothrombotic haematological conditions and HIV induced activation of some forms of systemic vasculitis [2]. Vasculitis un-related to infection and neoplasia rarely appears in the literature [3]. This case Identifies a HIV-infected patient presenting with recurrent episodes of ischaemic stroke along with a clinical and laboratory diagnosis of a vasculitic process of the CNS The findings indicated vasculitis of the central nervous system (CNS), but the workup failed to determine causes (infectious or neoplastic) for vasculitis other than HIV This report describes the clinical course and the radiologic findings.

\section{Case Details}

In 2000, a 17-year-old unmarried female patient was admitted with a two day history of right-sided body weakness, left-sided deviation of the mouth and drooling of saliva. History failed to indicate any risk factors for stroke and her family history supplied no further relevant information. On general physical examination, the patient exhibited pallor, oral candidiasis and extensive tenia cruris. Vital signs measured within normal limits. CNS examination revealed a decrease in muscle strength and tone on the right side with a power of 1/5. Exaggerated deep tendon reflexes with extensor plantar appeared on the right side. The provider reported normal limits for the remainder of the CNS examinations. Routine blood analysis showed no abnormalities except for haemoglobin of $9.2 \mathrm{mg} / \mathrm{dl}$. Cardiolipin and APLAS tested negative. Further diagnostic tests revealed positive HIV-1 antibodies by ELISA. Negative results came back for VDRL, HBsAg, Varicella, EBV and CMV titers. Toxoplasma and Cryptospordiasis also tested negative. The patient denied any high risk behavior associated with HIV infection. The patient's CD 4 count at that time measured $640 / \mu \mathrm{L}$. Blood and urine culture failed to show any growth. Echocardiography and ECG revealed normal results. CSF analysis showed lymphocytosis. Computed tomography (CT) of the brain revealed no abnormality. Contrast CT of the brain revealed vasculitic changes; carotid Doppler results fell within normal limits. Financial restrains prevented further medical evaluation. The patient underwent treatment with antifungals and physiotherapy for a month. The patient's condition improved and was discharged from the hospital once her muscle power improved to $3 / 5$.

In 2003, the patient presented again with symptoms of stroke, left hemi-paresis and right UMN fascial palsy. Central nervous system examination revealed a decrease in muscle strength and tone on the left side. Deep tendon reflexes appeared exaggerated on the left side with bilateral extensor plantar. The provider reported the rest of the CNS examination to be within normal limits. Routine blood analysis results tested within the normal limits. CD4 cell count measured 497/ $\mu \mathrm{L}$. The CT brain scan showed an old infarct in the left internal capsule. Symptomatic treatment ensued and the patient recovered with muscle power of $3 / 5$.

In 2007, patient presented again with left hemi paresis. The patient also had productive cough and the sputum examination tested positive for pulmonary tuberculosis. On physical examination, the patient exhibited pallor and oral candiasis. CNS examination revealed a decrease in muscle strength (0/5) and tone on left side. An exaggerated deep tendon reflex was observed on the left side with bilateral extensor plantar. The remainder of the CNS examination were within normal limits. Routine blood analyses fell within the normal limits. CD4 cell count measured $340 / \mu \mathrm{L}$. Computed tomography of the brain showed an old infarct in the left internal capsule. CSF analysis revealed lymphocytosis and no growth appeared on the blood culture. The patient underwent treatment with category-1 DOTS regimen. The patient's condition improved with physiotherapy, ecospirin and antifungals. At the time of discharge, the patient's sputum cleared the tuberculosis organisms. The muscle power on the affected side improved to $3 / 5$.

In 2010, the patient returned to the hospital again with left hemiparesis and right UMN fascial palsy. Patient also presented with

*Corresponding author: Shruti Shripad Hegde, Kasturba Medical College A-506, RNS Shanti Nivas, Post Yeshwanthpur, Tumkur Road, Bangalore-22, India, E-mail: shruti.hegde1587@gmail.com

Received March 14, 2013; Accepted April 08, 2013; Published April 15, 2013

Citation: Hegde SS, Ismail M, KulamarvaRama S, Abhishek H (2013) Recurrent Stroke as the First Manifestation in a Patient Infected with HIV- A Case Report. J AIDS Clin Res 4: 202. doi:10.4172/2155-6113.1000202

Copyright: $\odot 2013$ Hegde SS, et al. This is an open-access article distributed under the terms of the Creative Commons Attribution License, which permits unrestricted use, distribution, and reproduction in any medium, provided the original author and source are credited. 


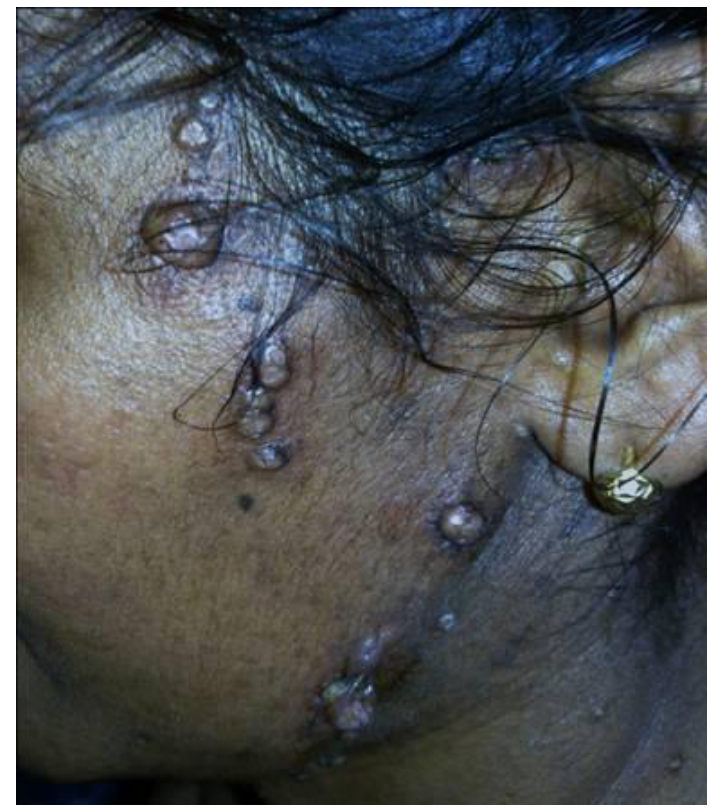

Figure 1: Extensive molluscum contagiosum
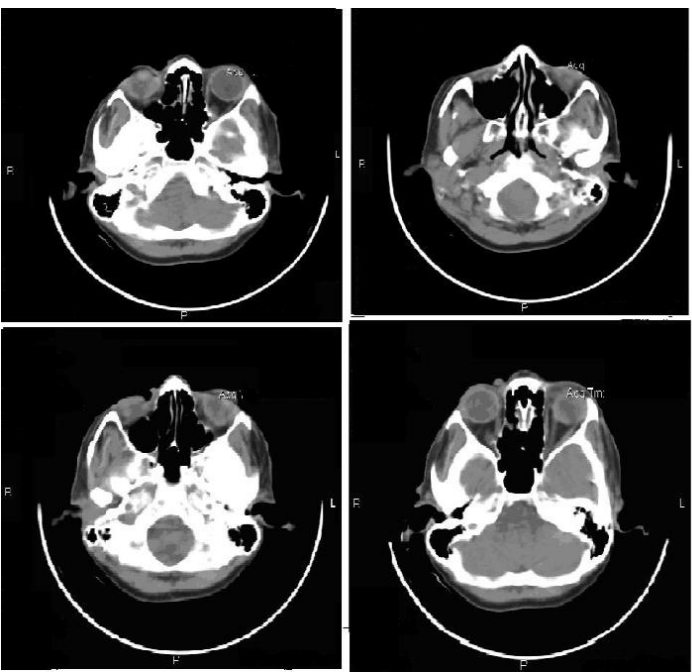

Figure 2: CT scan of the patient.

dysarthria, dysphagia, emotional lability and exaggerated jaw jerk. Patient diagnosed with pseudobulbar palsy. Extensive molluscum contagiosum lesions also found on the face neck and back (Figure 1). Patient exhibited oral candidiasis, but no meningism found. Muscle strength and tone diminished along with exaggerated deep tendon reflexes on the left side. Plantar reflexes werebilaterally extensors. No Romberg sign or cerebellar signs present. Routine blood analysis showed a raised ESR, a haemoglobin of $7 \mathrm{mg} / \mathrm{dl}$, anda CD4 cell count of $280 / \mu \mathrm{L}$. The measurement of the HIV plasma viral loadturned out to be not technically available at that time in our hospital. The CT brain scan showed bilateral old infarcts with gliosis in the basal-ganglia and the internal capsule with ischaemic changes visible in the left thalamus. The lateral and third ventricles appeared prominent for the age. Cortical sulci, bilateral sylvian fissures and basal cisterns also appeared prominent (Figure 2). Since the patient refused to undergo any further medical evaluation, a contrast enhanced CT scan and angiography had to be deferred. The patient underwent treatment with aspirin, bactrum DS, fluconazole, $\mathrm{KOH}$ mouth wash, trineurol, clopidogrel, haematinics and B-complex. The patient's condition improved and she went home from the hospital on day 45 .

\section{Discussion}

Recurrent stroke as a first manifestation of HIVremains extremely rare [3]. Nogueras et al. described a literature review on primary angitis of the CNS caused by HIV that led to recurrent strokes. The authors concluded that primary angitis leading to stroke represents a diagnosis of exclusion [4]. This case adds to the existing literature on the manifestation of HIV induced primary angitis causing recurrent stroke.

Studies have reported that individuals with stroke who are HIV positive are younger than patients with stroke who do not have HIV; this could be a result of the age of the population at risk of HIV infection or a sign that the mechanism of stroke in HIV is largely independent of classical vascular risk factors [5].

Mechanisms of stroke are variable in HIV-infected patients, with a relatively high incidence of vasculitis and hypercoagulability [6].

HIV-associated vasculopathy is used to describe various arterial changes associated with HIV infection. The term HIV-associated vasculopathy include any abnormality of the intracranial or extracranial cerebral blood vessels that results directly or indirectly from HIV infection, excluding vasculitis associated with opportunistic infection or neoplastic involvement of the vessels [5].

Although HIV-1 is less likely to be vasculotropic, the virus affects endothelial homoeostasis and function in important ways that could initiate and propagate atherogenesis [7]. The vascular endothelium is continually exposed to stimuli such as HIV-1-infected cells, freely circulating HIV-1 viruses, HIV-1 proteins that are released with host cell lysis or actively secreted, and viral-induced proinflammatory cytokines. All these factors potentially activate the endothelium, damaging and increasing its permeability, and thereby assisting leucocyte invasion into the vessel wall and chronic inflammation $[8,9]$. Infection of the endothelial cells by HIV or by other organisms, immune complex deposition, and impaired regulation of cytokines and adhesion molecules represent other factors hypothesized to play a role. It remains unclear whether HIV virus, viral structure or abnormal endothelial reaction bears responsibility for this phenomenon [4].

Assessment of the effect of HIV infection on stroke has public health relevance, particularly with respect to the increased frequency of stroke in regions of high HIV prevalence. From a practical clinical perspective, when physicians in all regions are managing a patient with HIV who has had a stroke, they need to know the extent to which the HIV infection and its treatment might affect the cause, clinical presentation, and management of the stroke, but they also need to consider that stroke might be the presenting feature of HIV infection in patients whose HIV status is not known.

HIV infection is a public health concern in mid-low income countries. Assessment of the effect of HIV infection on stroke is important. Physicians managing the HIV patient with stroke should have a thorough knowledge of the extent of the effect of HIV infection on stroke, its clinical presentation and management. They also need to consider that stroke might be the presenting feature of HIV infection in patients whose HIV status is not known [5].

In conclusion, recurrent stroke due to primary angitis may appear 
Citation: Hegde SS, Ismail M, KulamarvaRama S, Abhishek H (2013) Recurrent Stroke as the First Manifestation in a Patient Infected with HIV- A Case Report. J AIDS Clin Res 4: 202. doi:10.4172/2155-6113.1000202

Page 3 of 3

as the first manifestation in a HIV patient. This case reveals the value of evaluating HIV status in young people with stroke.

\section{References}

1. Ovbiagele B, Nath A (2011) Increasing incidence of ischemic stroke in patients with HIV infection. Neurology 76: 444-450.

2. Lee B, Anekthananon T, Poungvarin N, Nilanont $Y$ (2012) Etiology and risk factors of stroke in HIV-infected patients in Siriraj Hospital: a case-control study. J Med Assoc Thai 95 Suppl 2: S227-234

3. Nogueras C, Sala M, Sasal M, Viñas J, Garcia N, et al. (2002) Recurrent stroke as a manifestation of primary angiitis of the central nervous system in a patient infected with human immunodeficiency virus. Arch Neurol 59: 468-473.

4. Gorczyca I, Stanek M, Podlasin B, Furmanek M, Pniewski J (2005) Recurrent cerebral infarcts as the first manifestation of infection with the HIV virus. Folia Neuropathol 43: 45-49

5. Benjamin LA, Bryer A, Emsley HC, Khoo S, Solomon T, et al. (2012) HIV infection and stroke: current perspectives and future directions. Lancet Neuro 11: 878-890.

6. Ortiz G, Koch S, Romano JG, Forteza AM, Rabinstein AA (2007) Mechanisms of ischemic stroke in HIV-infected patients. Neurology 68: 1257-1261.

7. Chi D, Henry J, Kelley J, Thorpe R, Smith JK, et al. (2000) The effects of HIV infection on endothelial function. Endothelium 7: 223-242.

8. Tinkle BT, Ngo L, Luciw PA, Maciag T, Jay G (1997) Human immunodeficiency virus-associated vasculopathy in transgenic mice. J Virol 71: 4809-4814.

9. Hag AM, Kristoffersen US, Pedersen SF, Gutte H, Lebech AM, et al. (2009) Regional gene expression of LOX-1, VCAM-1, and ICAM-1 in aorta of HIV-1 transgenic rats. PLoS One 4: e8170. 